American Journal of Applied Sciences 6 (9): 1662-1668, 2009

ISSN 1546-9239

(C) 2009 Science Publications

\title{
Porcine Brain Extract Attenuates Memory Impairments Induced by Focal Cerebral Ischemia
}

\author{
${ }^{1}$ Jinatta Jittiwat, ${ }^{2}$ Jintanaporn Wattanathorn, ${ }^{2}$ Terdthai Tongun, \\ ${ }^{2}$ Supaporn Muchimapura and ${ }^{3}$ Chuleeratana Bunchonglikitkul \\ ${ }^{1}$ Department of Physiology and Graduate School (Neuroscience Program), \\ Faculty of Medicine and Graduate School, Khon Kaen University, Thailand, 40002 \\ ${ }^{2}$ Department of Physiology, Faculty of Medicine, Khon Kaen University, Thailand, 40002 \\ ${ }^{3}$ Thailand Institute of Scientific and Technological Research, Pathumthani, Thailand
}

\begin{abstract}
Problem statement: Stroke or cerebral ischemia has been recognized as one important problem worldwide. To date, the effectiveness of protective and therapeutic strategies against stroke is still very limited. Therefore, the development of novel strategy is required. Porcine brain is traditional believed to improve brain functions. Recent studies showed that the extract of porcine brain could protect against brain damage related to the oxidative stress, therefore, we hypothesized that it could protect against brain damage in stroke. Approach: To test the potential of porcine brain extract as the novel protective supplement against stroke, various doses of porcine brain extract at doses of 0.5 and $2.5 \mathrm{mg} \mathrm{kg}^{-1}$ b.w. were orally given to male Wistar rats, weighing 300-350 g, at the period of 14 days before and 21 days after the occlusion of right middle cerebral artery. Then, all rats were determined the neurological score, motor performance, cognitive function and brain infarct volume. Moreover, the possible neuroprotective mechanisms of the extract were also determined via the alteration of Malondialdehyde (MDA) or lipid peroxidation product and via the activities of scavenger enzymes including Superoxide Dismutase (SOD), Catalase (CAT) and Glutathione Peroxides (GPx). Results: The results showed that the low dose of porcine extract decreased the infarct volume and improved brain functions including neurological score, motor performance and memory deficit. In addition, it also decreased MDA but increased the activities of SOD, CAT and GPx. Conclusion: Our results suggested the potential role of porcine brain extract as neuroprotectant. The possible underlying mechanism appeared to be related to the enhanced activities of SOD, CAT and GPx which in turn resulted in the decrease MDA. Moreover, our findings may shed light on the pharmacologic basis for the clinical application of traditional Chinese medicine to protect against stroke.
\end{abstract}

Key words: Porcine brain extract, cerebral ischemia, stroke

\section{INTRODUCTION}

Cerebral ischemia or stroke has been recognized as one of the leading causes of death and disability in many countries ${ }^{[1]}$ including in Thailand ${ }^{[2]}$ due to the interruption of blood flow $^{[3]}$. Previous study demonstrated that the middle cerebral artery occlusion occurred in 10-15\% of stroke patients and resulted in the massive cerebral edema with raised intracranial pressure which finally progressed to coma and death ${ }^{[4]}$. Accumulating lines of evidence showed that free radicals contributed the important role on the brain damage after stroke and this damage could mitigate by substances possessing antioxidant ${ }^{[5]}$. Although, it is an important problem and produces an enormous impact on public health, no effective treatment has yet been found. Therefore, it is of interest to search for the novel effective strategy to prevent and attenuate the impairments induced by this condition.

Traditional Chinese Medicine (TCM) to improve the brain function has a long history. Porcine brain has been traditionally believed by people in some local areas of China and Thailand to improve brain functions and promote longevity. Previous studies had demonstrated that porcine brain derived peptide could protect brain against various insults including carbon monoxide $^{[6]}$ and glutamate ${ }^{[7]}$. Moreover, it could also decreased lipid peroxidation ${ }^{[8]}$ but increased the activities of various scavengering enzymes including superoxide dismutase, catalase ${ }^{[9]}$ and glutathione related

Corresponding Author: Jintanaporn Wattanathorn, Department of Physiology, Faculty of Medicine, Khon Kaen University, Thailand, 40002 Tel: 66-43-348394 
enzymes $^{[10]}$. Based on the potential neuroprotective effect of porcine brain derived peptide mentioned above, we hypothesized that the cytoplasmic fraction of porcine brain extract $(\mathrm{CF})$ could protect against brain damage induced by focal cerebral ischemia. To test whether cytoplasmic fraction of porcine brain extract can lead to the improvement of pathological and behavioral outcomes of focal cerebral ischemia, the effects of cytoplasmic fraction of porcine brain extract on cerebral infarction, neurological scores, postural tailhang test and the performance in Morris water maze test were determined. Moreover, in order to investigate the possible underlying mechanism of the cytoplasmic fraction of porcine brain extract, we also determined the effect of the extract on lipid peroxidation and scavenger enzymes including superoxide dismutase, catalase and glutathione peroxidase.

\section{MATERIALS AND METHODS}

Preparation of the cytoplasmic fraction of porcine brain extract: The standardized cytoplasmic fraction of porcine brain extract was kindly supported by Thailand Institute of Scientific and Technological Research, Phatumthani, Thailand. The descriptive processes were described as follows. The cytoplasmic fraction of porcine brain extract was prepared from young porcine's brain. The brain homogenate was homogenized with high speed homogenizer according to the following ratio; the brain tissue and buffer in a ratio of 1: 5-6 (weight/volume). Then, it was centrifuged at $12,500 \mathrm{rpm}$ for $1 \mathrm{~h}$, the supernatant fraction was separated and precipitated protein with acid. The extract was kept in airtight container at $4^{\circ} \mathrm{C}$ until used. The cytoplasmic fraction of porcine brain extract used in this study comprised of a mixture of amino acids consisting of glutamic acid and aspartic acid 13.80 and $11.03 \%$ respectively.

The extract was dissolved in $2 \%$ SCMC (sodium carboxymethyl cellulose) in order to obtain the desired concentration before administered orally via intragastric route once daily.

Animals: Male Wistar rats weighing 300-350 g were obtained from National Laboratory Animal Center, Salaya, Nakorn Pathom and were housed in group of 5 per cage in standard metal cages at $22 \pm 2^{\circ} \mathrm{C}$ on $10: 14$ $\mathrm{h}$ light-dark cycle. All animals were given access to food and water ad labium. The experiments were performed to minimize animal suffering in accordance with the internationally accepted principles for laboratory use and care of European Community (EEC directive of 1986; 86/609/EEC).
The experimental protocols were approved by the Institutional Animal Care and Use Committee.

Experimental protocol: In order to induce focal cerebral ischemia, all rats in this study were occluded the right Middle Cerebral Artery Occlusion (MCAO). Rats were randomly divided into various groups as described following: (1) Vehicle + MCAO; (2) Aricept + MCAO (positive control); (3) Piracetam + MCAO (positive control); (4) Vitamin $\mathrm{C}+\mathrm{MCAO}$ (positive control); (5) $\mathrm{CF}$ (0.5 mg kg-1 b.w.) + MCAO and (6) CF1 (2.5 $\mathrm{mg} \mathrm{kg}^{-1}$ b.w.) + MCAO. The animals in group 2 were treated with Aricept, a cholinesterase inhibitor which used as standard treatment of dementia at dose of $1 \mathrm{mg} \mathrm{kg}-1$ whereas those in group 3 were treated with piracetam (250 $\mathrm{mg} \mathrm{kg}^{-1}$ b.w.), a standard drug used to improve cerebral blood flow and we also used vitamin C (100 mg kg-1 b.w.), a well known antioxidant, as positive control. The animals in the last 2 groups were treated with CF at doses of 0.5 and $2.5 \mathrm{mg} \mathrm{kg}^{-1}$ b.w., respectively. These two doses of the extract were selected based on our pilot study which demonstrated the effect of the extract on the central nervous system. All animals were treated with vehicle or positive control or $\mathrm{CF}$ extract at a period of 2 weeks before and 3 weeks after right Middle Cerebral Artery Occlusion (MCAO).

Focal cerebral ischemia induction: All rats were fasted for $12 \mathrm{~h}$ but were allowed free access to water before surgery. Anesthesia was induced with intraperitoneal injection of thiopental sodium at dose of $50 \mathrm{mg} \mathrm{kg}^{-1}$ bw. Focal cerebral ischemia was induced by modified previous method described by Longa et al. Briefly, the right common carotid artery and the right external carotid artery were exposed through a ventral midline neck incision and were ligated proximally. A silicone coated nylon monofilament (4-0) suture (USS $\mathrm{DG}^{\mathrm{TM}}$ sutures; Tyco Healthcare group LP, Connecticut, USA) with its tip rounded by heating near a flame was inserted through an arteriectomy in the common carotid artery just below the carotid bifurcation and then advanced into the internal carotid artery approximately 17-18 $\mathrm{mm}$ distal to the carotid bifurcation until a mild resistance was felt. Occlusion of the origins of the anterior cerebral artery, the middle cerebral artery and the posterior communicating artery was thereby achieved. Then, the wound was sutured, the rats were returned to their cages with free access to food and water. The incision sites were infiltrated with $10 \%$ PovidoneIodine Solution for anti-septic postoperative care.

Morris water maze test: The water maze consisted of a metal pool $(170 \mathrm{~cm}$ in diameter $\times 58 \mathrm{~cm}$ tall $)$ filled 
with tap water $\left(25^{\circ} \mathrm{C}, 40 \mathrm{~cm}\right.$ deep) divided into 4 quadrants. In the center of 1 quadrant was a removable escape platform below the water level and covered with a nontoxic milk powder. The pool was divided into 4 quadrants (NE, NW, SE and SW) by two imaginary lines crossing the center of the pool. For each animal, the location of invisible platform was placed at the center of one quadrant and remained there throughout training. The rats must memorize the platform location in relation to various environmental cues because there was nothing directly shown the location of the escape platform in and outside the pool. Therefore, the placement of the water tank and platform were the same in all acquisition trials. Each rat was gently placed in the water facing the wall of the pool from one of the four starting points $(\mathrm{N}, \mathrm{E}, \mathrm{S}$, or $\mathrm{W})$ along the perimeter of the pool and the animal was allowed to swim until it found and climbed onto the platform. During training session, the subject was gently placed on the platform by the experimenter when it could not reach the platform in $60 \mathrm{~s}$. In either case, the subject was left on the platform for $15 \mathrm{~s}$ and removed from the pool. The time for animals to climb on the hidden platform was recorded as escape latency. In addition to the acquisition test, the determination of retention memory was carried out on the next day. According to this test, the platform was removed and the animals were placed into the water maze for $60 \mathrm{~s}$. The retention of memory or the time that the animal spent to swim around the previous location of platform before removing the platform on the test occurring in the next day was also recorded. It has been postulated that if the spatial memory of the rat for the trained platform location is accurate, the rat will swim to the platform location and search around the exact location. Therefore, the more accurate the spatial memory is, the greater the number of times rat swim across the trained platform. In each trial, the animal was quickly dried with towel before being returned to the cage. All tests in Morris water maze test were carried out within $30 \mathrm{~min}$ after the oral administration of the substances.

Determination of infarct volume: All animals were killed $24 \mathrm{~h}$ after occlusion and the brain was removed and sectioned at $2 \mathrm{~mm}$ thick. Sections were immersed in $2 \% \mathrm{TTC}(2,3,5$-triphenyltetrazolium chloride) for $30 \mathrm{~min}$ at $37^{\circ} \mathrm{C}$. Images of stained sections were digitized and infarction volumes were determined using an indirect method ${ }^{[11]}$.

Determination of neurological score: The neurological deficit score was performed by a single experimenter, who was blinded to the experimental groups according to method of Bederson et al. ${ }^{[12]}$. The deficit was graded on a scale of $0-5$ as follow: Grade 0 , no spontaneous activity, (Grade 1) Spontaneous circling; (Grade 2) Circling if pulled by tail; (Grade 3) lowered resistance to lateral push without circling; (Grade 4) Contralateral forelimb flexion; (Grade 5) No apparent deficit.

Biochemical assay: Rats were perfused with cold saline solution to get rid of the blood from the brain tissue, then rapidly removed and stored at $70^{\circ} \mathrm{C}$ until used.

Glutathione Peroxidase (GPx) level: Glutathione peroxidase activity was measured using spectrophotometric method ${ }^{[13]}$. This activity was determined using $\mathrm{H}_{2} \mathrm{O}_{2}$ as the substrate and $\mathrm{GR}$ and NADPH as enzymatic and non-enzymatic indicators, respectively.

Catalase activity determination: Catalase activity in the supernatant was measured by recording the rate of decrease in $\mathrm{H}_{2} \mathrm{O}_{2}$ absorbance at $240 \mathrm{~nm}^{[14]}$. The activity of catalase was expressed as $\mu \mathrm{mol} \mathrm{H}_{2} \mathrm{O}_{2} \mathrm{~min}^{-1} \mathrm{mg}^{-1}$ protein.

Superoxide Dismutase (SOD) activity determination: SOD activity was measured in supernatant ${ }^{[15]}$. SOD activity was expressed in terms of $\mathrm{U} \mathrm{mg}^{-1}$ protein, where 1 Unit was defined as the amount of enzyme that inhibited the optical density at $560 \mathrm{~nm}$ of chromogen production by $50 \%$ under the above assay conditions. Results were expressed as $\mathrm{U} \mathrm{min}^{-1} \mathrm{mg}^{-1}$ protein.

Statistical analysis: Data were presented as mean \pm Standard Error of Mean (SEM). The analysis was performed using one-way Analysis Of Variance (ANOVA), followed by Dunnett's test. All statistical results were considered significant if $\mathrm{p}$-value $<0.05$.

\section{RESULTS}

Effect of CF1 extract on cognitive function: The effect of CF1 extract on cognitive function was determined using Morris water maze test. Before stroke, Aricept, Piracetam, Vitamin C and CF1 extract at dose $0.5 \mathrm{mg} \mathrm{kg}^{-1}$ b.w. significantly decreased the escape latency in Morris water maze test as compared to the vehicle treated group whereas the significant effect of CF1 at dose $2.5 \mathrm{mg} \mathrm{kg}^{-1}$ b.w. was observed at single dose administration. Aricept, Piracetam, Vitamin $\mathrm{C}$ and $\mathrm{CF} 1$ at both doses could also significantly decreased the escape latency time in MCAO rat after 1 week of MCAO and the significant was observed until the end of the experiment (Fig. 1). 


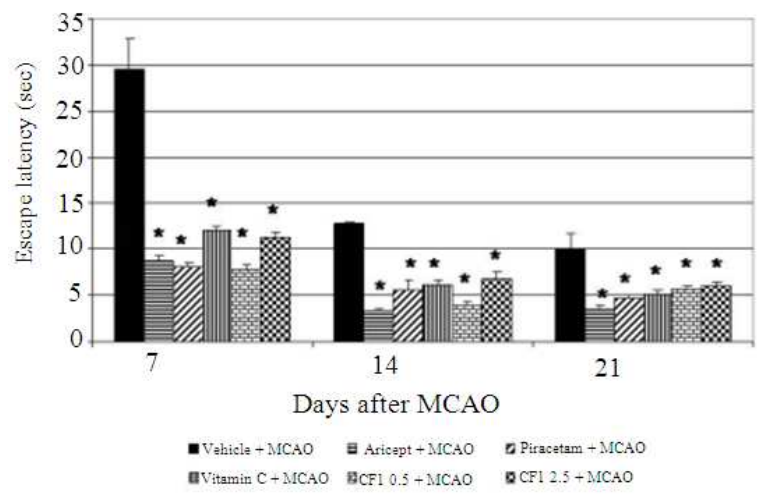

Fig. 1: Effect of aricept, vitamin C, piracetam and various doses of cytoplasmic fraction of porcine brain extract ( $\mathrm{CF} 1)$ on escape latancy in Morris wter maze test. Values are the mean \pm SEM $(\mathrm{n}=6) *$ : p-value $<0.05$ as compared with vehicle plus MCAO

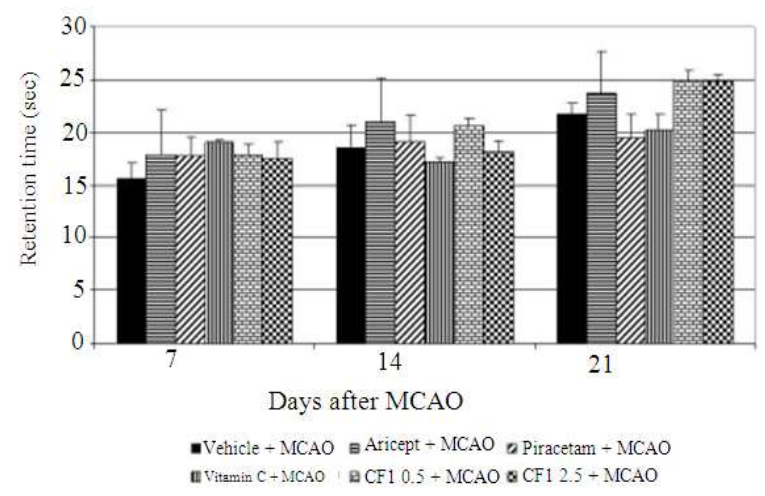

Fig. 2: Effect of aricept, vitamin C, piracetam and various doses of cytoplasmic fraction of porcine brain extract (CF1) on retention time in Morris water maze test. Values given are the mean \pm SEM $(n=6)$

Effect of CF1 extract on motor function: The effect of CF1 extract on motor function was evaluated using neurological score (Fig.2). 7 days after right middle cerebral artery occlusion Aricept treated groups significantly increased the neurological score compared to vehicle treated group. Aricept, CF1 extract at dose $0.5 \mathrm{mg} \mathrm{kg}{ }^{-1}$ b.w. were observed the significantly improvement of neurological score at 14 after right middle cerebral artery occlusion whereas Piracetam was observed the significantly improvement of neurological score at 21 days after right middle cerebral artery occlusion as compared to vehicle treated group (Table $1)$.
Table 1: Effect of aricept, vitamin C, piracetam and various doses of cytoplasmic fraction of porcine brain extract (CF1) on motor function using neurological score. Values given are the mean \pm SEM $(n=6)$

\begin{tabular}{|c|c|c|c|c|}
\hline \multirow{3}{*}{$\begin{array}{l}\text { Duration } \\
\text { Groups day }^{-1}\end{array}$} & \multicolumn{4}{|c|}{ Neurological score } \\
\hline & \multicolumn{3}{|c|}{ After MCAO } & \multirow[b]{2}{*}{ Day 21} \\
\hline & Day 0 & Day 7 & Day 14 & \\
\hline Vehicle + MCAO & $5.00 \pm 0.00$ & $2.40 \pm 0.24^{\#}$ & $3.20 \pm 0.20^{\#}$ & $3.60 \pm 0.25^{\sharp}$ \\
\hline Aricept + MCAO & $5.00 \pm 0.00$ & $3.60 \pm 0.24^{\# *}$ & $4.20 \pm 0.20^{\# *}$ & $4.80 \pm 0.20$ \\
\hline Vitamin $\mathrm{C}+\mathrm{MCAO}$ & $5.00 \pm 0.00$ & $3.20 \pm 0.37^{\#}$ & $3.80 \pm 0.20^{\#}$ & $4.20 \pm 0.20^{\#}$ \\
\hline Piracetam + MCAO & $5.00 \pm 0.00$ & $3.20 \pm 0.37^{\#}$ & $3.40 \pm 0.25^{\#}$ & $4.60 \pm 0.40^{*}$ \\
\hline CF1 $0.5+\mathrm{MCAO}$ & $5.00 \pm 0.00$ & $3.20 \pm 0.37^{\#}$ & $4.00 \pm 0.32^{\# *}$ & $4.40 \pm 0.25$ \\
\hline CF1 $2.5+\mathrm{MCAO}$ & $5.00 \pm 0.00$ & $2.80 \pm 0.37^{\#}$ & $3.20 \pm 0.25^{\#}$ & $4.00 \pm 0.31^{\#}$ \\
\hline
\end{tabular}

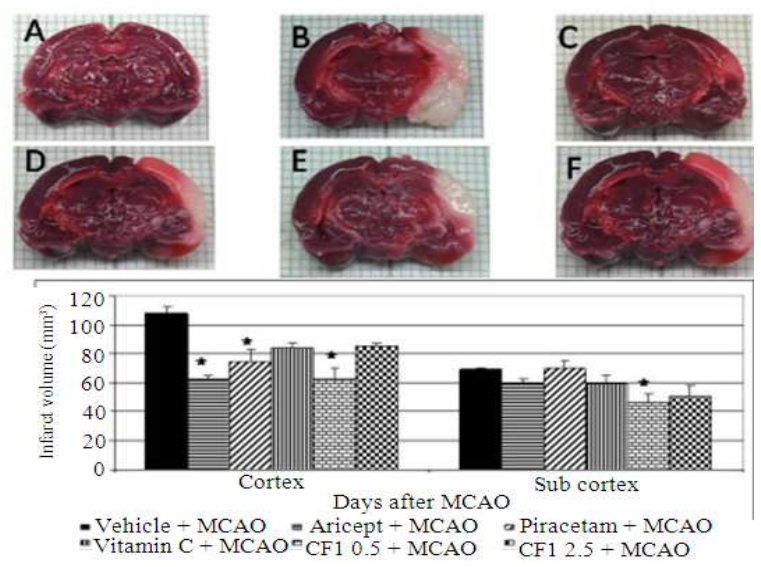

Fig. 3: Effect of aricept, vitamin C, piracetam and various doses of cytoplasmic fraction of porcine brain extract $(\mathrm{CF} 1)$ on brain infarct volume. Brain infarct volume was determined using TTC staining. Values given are the mean \pm SEM $(\mathrm{n}=6) *$ : p-value $<0.05$ as compared with vehicle plus MCAO

Effect of CF1 extract on cerebral infarction: Infarct volumes were measured using an indirect method in the $2 \mathrm{~mm}$ thick coronal brain sections which were stained with 2\% TTC (2,3,5-triphenyltetrazolium chloride). Aricept, Piracetam and CF1 extract at dose $0.5 \mathrm{mg} \mathrm{kg}^{-1}$ b.w. significantly decreased the infarct volume in cortex whereas CF1 extract at dose $0.5 \mathrm{mg} \mathrm{kg}^{-1}$ b.w. significantly decreased the infarct volume in subcortex region (Fig. 3). The infarct volume of CF1 extract at dose $0.5 \mathrm{mg} \mathrm{kg}^{-1}$ b.w. treated group was decreased to $62.82 \pm 7.63 \mathrm{~mm}^{3}, 46.31 \pm 6.15 \mathrm{~mm}^{3}$ in cortex and sub cortex, respectively.

Effect of CF1 extract on superoxide dismutase activity: Superoxide dismutase activity was estimated in the ipsilateral hemisphere of the ischemic animal after $24 \mathrm{~h}$ of occlusion. 


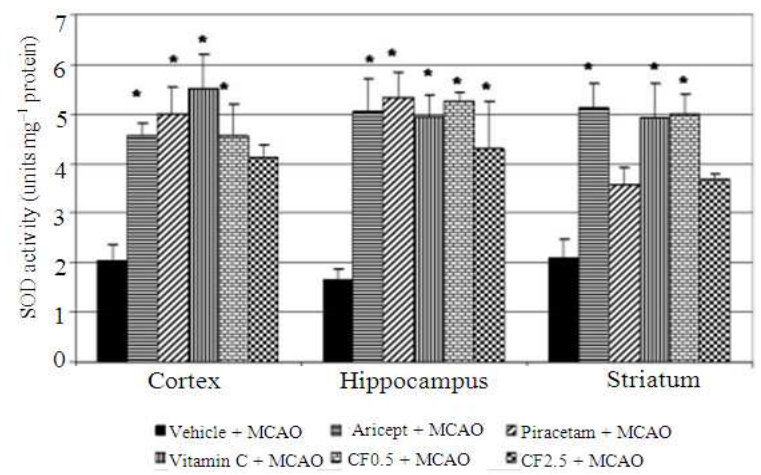

Fig. 4: Effect of aricept, vitamin C, piracetam and various doses of cytoplasmic fraction of porcine brain extract (CF1) on the superoxide dismutase (SOD) activity in ceriberal cortex, hippocampus and striatum. Values given are the mean \pm SEM $(\mathrm{n}=6) *$ : p-value $<0.05$ as compared with vehicle plus MCAO

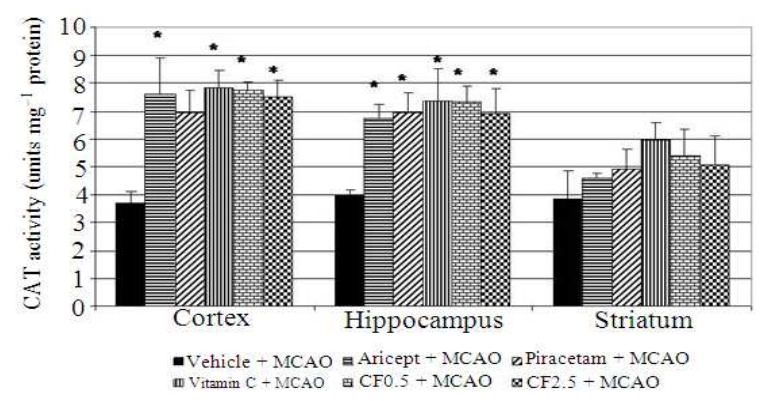

Fig. 5: Effect of aricept, vitamin C, piracetam and various doses of cytoplasmic fraction of porcine brain extract (CF1) on the catalase (CAT) activity in ceriberal cortex, hippocampus and striatum. Values given are the mean \pm SEM $(\mathrm{n}=6) *$ : p-value <0.05 as compared with vehicle plus MCAO

Ischemic stroke induced decrease in SOD activity was reverted by Aricept, Piracetam, Vitamin C and CF1 extract at dose $0.5 \mathrm{mg} \mathrm{kg}^{-1}$ b.w. in cortex, hippocampus and striatum whereas CF1 extract at dose $2.5 \mathrm{mg} \mathrm{kg}^{-1}$ b.w. could reverted in hippocampus (Fig. 4).

Effect of CF1 extract on catalase activity: Catalsae activity was estimated in the ipsilateral hemisphere of the ischemic animal after $24 \mathrm{~h}$ of occlusion. The catalase activity was significantly elevated in Aricept, Vitamin $\mathrm{C}$ and $\mathrm{CF} 1$ both doses treated animals subjected to middle cerebral artery occlusion in cortex area and hippocampus whereas Piracetam significantly elevated the catalase activity only in hippocampus as compared to vehicle treated group (Fig. 5).

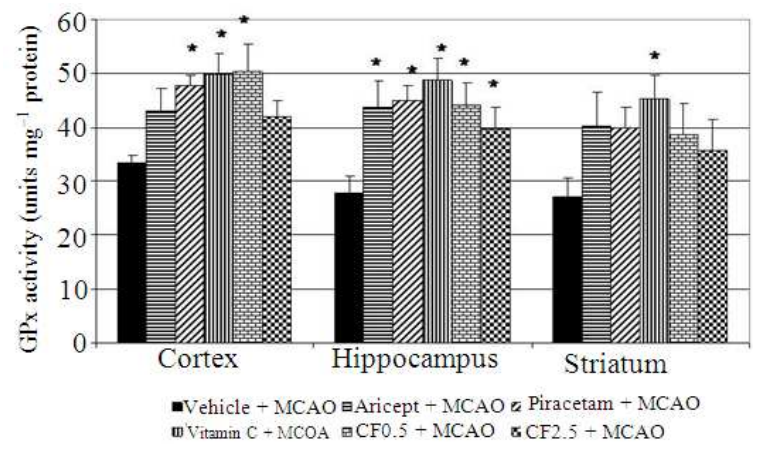

Fig. 6: Effect of aricept, vitamin C, piracetam and various doses of cytoplasmic fraction of porcine brain extract (CF1) on the activity of glutathoine peroxide (GSH-Px) in ceriberal cortex, hippocampus and striatum. Values given are the mean \pm SEM $(n=6) *$ : p-value $<0.05$ as compared with vehicle plus MCAO

Effect of CF1 extract on glutathione peroxidase activity: Glutathione peroxidase activity was estimated in the ipsilateral hemisphere of the ischemic animal after $24 \mathrm{~h}$ of occlusion. The glutathione peroxidase activity was significantly elevated in all group of treatment in hippocampus as compared to vehicle treated group. Piracetam, Vitamin C and CF1 $0.5 \mathrm{mg} \mathrm{kg}^{-1}$ b.w. treated animals subjected to middle cerebral artery occlusion were significantly increased the glutathione peroxidase activity in cortex area whereas in striatum the glutathione peroxidase activity was significantly increased in Vitamin C treated group as compared to vehicle treated group (Fig. 6).

\section{DISCUSSION}

The present study clearly demonstrated that the cytoplasmic fraction of porcine brain extract at dose of $0.5 \mathrm{mg} \mathrm{kg}^{-1}$ b.w. ameliorated the volume of cerebral infarction induced by focal cerebral ischemia in both cortical and subcortical areas. The improvement of performance in Morris water maze test was observed after 1 week or 7 days of MCAO and this improvement was still observed throughout the experimental period. However, the improvement of neurological score and the postural tail hang-test were observed at 2 and weeks after MCAO. The changes in both behavior and neurological score mentioned earlier further confirmed that the cytoplasmic fraction of porcine brain extract was effective in reducing the stroke damage induced by focal cerebral ischemia via MCAO. These data suggested that the increase exposure time to the extract resulted in the more improvement of brain functions. 
The possible explanation might be associated with the more accumulation of active ingredients of the extract in various target areas. However, this still required further investigation. In contrast to the effect of exposure time, no significant change was observed when the concentration of the extract was further increased to $2.5 \mathrm{mg} \mathrm{kg}^{-1}$ b.w. Although, the precise change of this phenomenon was still beyond the scope of this study, we did suggest that it might be related with the masking effect of various ingredients in the extract which could possibly mitigate the effect of active ingredient. In addition, it was also possible that the effect had already been saturated. However, due to the limitation of the extract in this batch, we could have only 2 doses of the extract. Therefore, further researches are still essential to explore this issue. The data from this study also indirectly pointed out the different in vulnerability of various brain areas to the extract. It was found that the significant improvement of water maze performance appeared before other changes, therefore, it indirectly suggested the highest vulnerability of hippocampus. Recent findings showed that hippocampus was the area which had high plasticity and contained high capability of neurogenesis ${ }^{[16]}$. Therefore, we suggested that the highest vulnerability to the cytoplasmic fraction of porcine brain extract might be due to this property of hippocampus.

Numerous lines of evidence showed that the free radicals contributed the important role in the pathophysiology of brain damage after cerebral ischemia and this impairment could be mitigated by the antioxidant by increasing the activities of scavenger enzymes $^{[5]}$. Previous studies demonstrated that the upregulation of the scavenger enzymes could reduce both global and focal ischemic injury ${ }^{[17,18]}$. Our results also showed that the extract could increase the activity of superoxide dismutase in cerebral cortex, hippocampus and striatum. However, the increase of catalase and glutathione peroxidase were observed only in cerebral cortex and hippocampus. Therefore, our findings are in agreement with previous studies. Taken together, these data suggested that the neuroprotective effect of the cytoplasmic fraction of porcine brain extract might occur partly via the increase scavenger enzymes activities resulting in the decrease oxidative stress or free radical. The superoxide dismutase appeared to contribute more role than other enzymes such as catalase and glutathione peroxidase. The possible explanation for this change might be associated with the increase superoxide anion production in focal cerebral ischemia ${ }^{[19]}$.

\section{CONCLUSION}

In conclusion, the cytoplasmic fraction of porcine brain extract could effectively mitigate the ischemiainduced pathological and behavioral changes and cognitive impairment. Pharmacological characteristics of the extract require further investigated. However, efficacy of the extract is consistent with the improvement of the scavenger enzymes and provide the valid evidence to support the potential of the cytoplasmic fraction of porcine brain extract as a neuroprotectant.

\section{REFERENCES}

1. Bonita, R., S. Mendis, T. Truelsen, J. Bogousslavsky, J. Toole and F. Yatsu, 2004. The global stroke initiative. Lancet Neurol., 3: 391-393. http://www.ncbi.nlm.nih.gov/pubmed/15207791

2. Khor, G.L., 200. Cardiovascular epidemiology in the Asia-pacific region. Asia Pac. J. Clin. Nutr., 10: 76-80.

http://www.ncbi.nlm.nih.gov/pubmed/11710361

3. Feigin, V.L., C.M. Lawes, D.A. Bennett and C.S. Anderson, 2003. Stroke epidemiology: A review of population-based studies of incidence, prevalence and case-fatality in the late 20th century. Lancet Neurol., 2: 43-53. http://www.ncbi.nlm.nih.gov/pubmed/12849300

4. Hacke, W., S. Schwab, M. Horn, M. Spranger, M. De Georgia and R. Von Kummer, 1996. 'Malignant' middle cerebral artery territory infarction: Clinical course and prognostic signs. Arch. Neurol., 53: 309-315. http://www.ncbi.nlm.nih.gov/pubmed/8929152

5. Ullegaddi, R., H.J. Powers and S.E. Gariballa, 2006. Antioxidant supplementation with or without B-group vitamins after acute ischemic stroke: A randomized controlled trial. JPEN J. Parenter. Enteral. $\quad$ Nutr., $\quad 30$ : 108-114. http://grande.nal.usda.gov/ibids/index.php?mode2= detail\&origin=ibids_references\&therow $=790612$

6. Koroleva, V.I., O.S. Korolev, V. Mares, E. Pastalkova and J. Bures, 1999. Hippocampal damage induced by carbon monoxide poisoning and spreading depression is alleviated by chronic treatment with brain derived polypeptides. Brain Res., 816: 618-627. http://www.ncbi.nlm.nih.gov/pubmed/9878887

7. Hutter-Paier, B., E. Grygar and M. Windisch, 1996. Death of cultured telencephalon neurons induced by glutamate is reduced by the peptide derivative Cerebrolysin. J. Neural. Transm. Suppl., 47: 267-273.

http://www.ncbi.nlm.nih.gov/pubmed/8841972 
8. Patockova, J., M. Krsiak, P. Marhol and E. Tumova, 2003. Cerebrolysin inhibits lipid peroxidation induced by insulin hypoglycemia in the brain and heart of mice. Physiol. Res., 52: 455-460. http://www.ncbi.nlm.nih.gov/pubmed/12899658

9. Gonzalez, C., G. Farias and R.B. Maccioni, 1998. Modification of tau to an Alzheimer's type protein interferes with its interaction with microtubules. Cell. Mol. Biol. (Noisy-le-grand), 44: 1117-1127. http://www.ncbi.nlm.nih.gov/pubmed/9846894

10. Cruz, R., L. Francis, C.M. Diaz-Suarez and M.E. Gonzalez-Fraguela, 1998. Short-term effects of septo-hippocampal pathway transsection and cerebrolysin effects on glutathione-related enzymes in the rat brain. Rev. Neurol., 26: 551-554. http://www.ncbi.nlm.nih.gov/pubmed/9796003

11. Swanson, R.A., M.T. Morton, G. Tsao-Wu, R.A. Savalos, C. Davidson and F.R. Sharp, 1990. A semiautomated method for measuring brain infarct volume. J. Cereb. Blood Flow Metab., 10: 290-293.

http://www.ncbi.nlm.nih.gov/pubmed/1689322

12. Bederson, J.B., L.H. Pitts, M. Tsuji, M.C. Nishimura, R.L. Davis and H. Bartkowski, 1986. Rat middle cerebral artery occlusion: Evaluation of the model and development of a neurologic examination. Stroke, $\quad 17$ : 472-476. http://www.ncbi.nlm.nih.gov/pubmed/3715945

13. Flohe, L. and W.A. Gunzler, 1984. Assays of glutathione peroxidase. Methods Enzymol., 105: 114-121. http://www.ncbi.nlm.nih.gov/pubmed/6727659

14. Aebi, H., S.R. Wyss, B. Scherz and F. Skvaril, 1974. Heterogeneity of erythrocyte catalase II. Isolation and characterization of normal and variant erythrocyte catalase and their subunits. Eur. J. Biochem., 48: 137-145. http://www.ncbi.nlm.nih.gov/pubmed/4141308
15. Kakkar, P., B. Das and P.N. Viswanathan, 1984. A modified spectrophotometric assay of superoxide dismutase. Ind. J. Biochem. Biophys., 21: 130-132. http://www.ncbi.nlm.nih.gov/pubmed/6490072

16. Eriksson, P.S., E. Perfilieva, T. Bjork-Eriksson, A.M. Alborn, C. Nordborg, D.A. Peterson and F.H. Gage, 1998. Neurogenesis in the adult human hippocampus. Nat. Med., 4: 1313-1317. http://www.nature.com/nm/journal/v4/n11/abs/nm1 198_1313.html

17. Sheng, H., R.D. Bart, T.D. Oury, R.D. Pearlstein, J.D. Crapo and D.S. Warner, 1999. Mice overexpressing extracellular superoxide dismutase have increased resistance to focal cerebral ischemia. Neuroscience, 88: 185-191. http://www.ncbi.nlm.nih.gov/pubmed/10051199

18. Yang, G., P.H. Chan, J. Chen, E. Carlson, S.F. Chen, P. Weinstein, C.J. Epstein and H. Kamii, 1994. Human copper-zinc superoxide dismutase transgenic mice are highly resistant to reperfusion injury after focal cerebral ischemia. Stroke, 25: $165-170$. http://www.ncbi.nlm.nih.gov/pubmed/8266365

19. Peters, O., T. Back, U. Lindauer, C. Busch, D. Megow, J. Dreier and U. Dirnagl, 1998. Increased formation of reactive oxygen species after permanent and reversible middle cerebral artery occlusion in the rat. J. Cereb. Blood Flow Metab., 18: 196-205. http://www.ncbi.nlm.nih.gov/pubmed/9469163 